\title{
Non-linear charge and energy dynamics of an adiabatically driven interacting quantum dot
}

\author{
Javier I. Romero, ${ }^{1}$ Pablo Roura-Bas, ${ }^{2}$ Armando A. Aligia, ${ }^{3}$ and Liliana Arrachea ${ }^{1}$ \\ ${ }^{1}$ International Center for Advanced Studies, ECyT-UNSAM, \\ Campus Miguelete, 25 de Mayo y Francia, 1650 Buenos Aires, Argentina \\ ${ }^{2}$ Dpto de Física, Centro Atómico Constituyentes, Comisión Nacional de Energía Atómica, CONICET, Buenos Aires, Argentina \\ ${ }^{3}$ Centro Atómico Bariloche and Instituto Balseiro, Comisión Nacional de Energía Atómica, CONICET, 8400 Bariloche, Argentina
}

(Dated: September 19, 2018)

\begin{abstract}
We formulate a general theory to study the time-dependent charge and energy transport of an adiabatically driven interacting quantum dot in contact to a reservoir for arbitrary amplitudes of the driving potential. We study within this framework the Anderson impurity model with a local ac gate voltage. We show that the exact adiabatic quantum dynamics of this system is fully determined by the behavior of the charge susceptibility of the frozen problem. At $T=0$, we evaluate the dynamic response functions with the numerical renormalization group (NRG). The time-resolved heat production exhibits a pronounced feature described by an instantaneous Joule law characterized by an universal resistance quantum $R_{0}=h /\left(2 e^{2}\right)$ for each spin channel. We show that this law holds in non-interacting as well as in the interacting system and also when the system is spin-polarized. In addition, in the presence of a static magnetic field, the interplay between many-body interactions and spin polarization leads to a non-trivial energy exchange between electrons with different spin components.
\end{abstract}

PACS numbers: 73.23.-b, 73.63.Kv,72.15.Qm

\section{INTRODUCTION}

The generation of electron currents by locally applying timedependent voltages in coherent conductors is a topic of intensive research activity for some years now. Any mechanism to be implemented with this goal is accompanied by energy dissipation.

Quantum capacitors are prominent experimental realizations of these systems $\frac{1-3}{-\underline{3}}$ They were introduced by Büttiker, Thomas and Prêtre as quantum equivalents of the classical linear RC circuits, $\stackrel{4}{-6}$ by assuming a small amplitude of the driving voltage. The corresponding ac complex impedance depends on the driving frequency, the capacitance of the quantum dot and the resistance of the circuit. In the original theory, $\underline{\underline{4}} \underline{\underline{6}}$ transport coherence is assumed along the full setup, and the only resistive element is the contact, which results in a quantized electron relaxation resistance $R_{q}=R_{0} / N_{c}$ where $N_{c}$ is the number of transport channels and $R_{0}=h /\left(2 e^{2}\right)$, is the resistance quantum. The universality of this resistance remains robust in the low frequency regime upon adding electronelectron interactions in the quantum dot provided that the system behaves as a Fermi liquid (FL) ${ }^{7-11}$

While in some experiments the driving amplitudes were within the range of linear response theory, $\frac{1}{1}$ further experimental ${ }^{2.3}$ and theoretica ${ }^{12-15}$ contributions focused on quantum capacitors as single-electron sources, implying large amplitudes. In Ref. 12 a theory for the regime of large amplitudes was proposed for non-interacting systems. The effect of many-body interactions was later considered within perturbation theory,,$\frac{16}{,}$ mean-field approximations,$\frac{17}{,}$ and exact approaches valid in the large-transparency limit $\frac{18}{\underline{1}}$ One of the goals of the present contribution is to study the low-frequency non-linear regime while fully taking into account many-body interactions and spin-polarization effects caused by external magnetic fields.

The setup consists of a quantum dot driven by a gate voltage $V_{g}(t)$ and connected to an electron reservoir, as sketched in
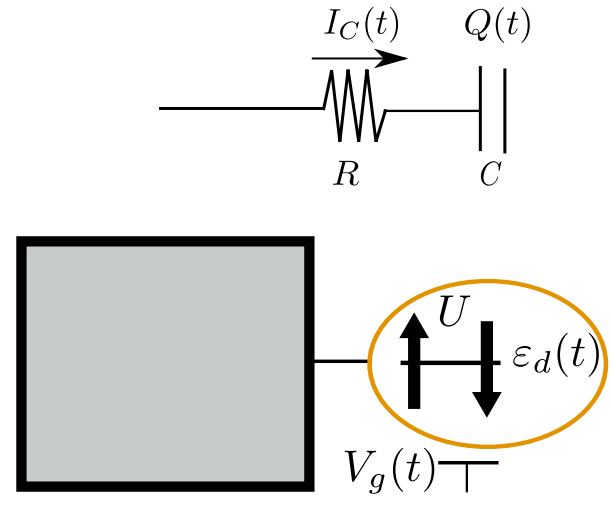

FIG. 1. (Color online) Sketch of the setup. A quantum dot described by a single electron level with Coulomb interaction $U$ and is driven by an ac gate voltage $V_{g}(t)=V_{0} \sin (\Omega t)$ and is connected to a normal lead. Top: representation of the setup in terms of a resistance connected in series with a capacitor.

Fig.1. We focus on the so called adiabatic regime where the time scale associated to a variation of $V_{g}(t)$ is much larger than the characteristic time scale for the dynamics of the electrons inside the quantum dot.

As mentioned before, in linear response, it is usual to represent this setup in terms of a resistance in series with a capacitor, as sketched at the top of Fig. 1 1 -11 In this paper, we show that this representation with $R=R_{q}$ is also sound to describe the adiabatic dynamics of the interacting system without magnetic field beyond linear response. In the case of a magnetic field applied at the quantum dot, we analyze the setup in the context of the circuit sketched in Fig. 2, where each spin channel is regarded as a branch of a circuit with a capacitance in series with a resistance accounting for a total voltage drop $V_{g}(t)$. For the quantum dot without many-body interactions, we show that the resistance per spin channel is $R_{0}$, while we argue that for the interacting quantum dot with magnetic field, 
the charge dynamics cannot be properly represented by this circuit.

The quantum dot-reservoir system dissipates the energy received from the ac source in the form of heat that flows into the reservoir. For a non-interacting quantum dot connected to a single-channel reservoir at zero temperature and for low frequency but arbitrary amplitude of the driving potential, the time-dependent rate for the dissipation of energy was found to obey an instantaneous Joule law (IJL), with the universal resistance $R_{0}, \frac{19,21}{1}$

$$
P_{\text {Joule }}(t)=R_{0} \sum_{\sigma}\left[I_{C, \sigma}(t)\right]^{2},
$$

where $I_{C, \sigma}(t)$ is the instantaneous charge current of electrons with spin $\sigma$ flowing from the quantum dot to the reservoir. Here we investigate to what an extent the Coulomb interaction at the quantum dot affects this picture. We analyze this ingredient in the framework of the Anderson impurity model. One of the scenarios in this context is the Kondo effect, which takes place below the so called Kondo temperature $T_{K}$ when the quantum dot is strongly connected to the reservoirs and occupied by an odd number of electrons ${ }^{23}$ The electrons of the reservoir and the effective spin 1/2 localized at the quantum dot form a many-body singlet state. Other scenario is the Coulomb blockade, according to which it is necessary to overcome the energy of the Coulomb interaction to introduce an additional electron in the quantum dot once it is already filled with one electron. In all the regimes, the single impurity Anderson model behaves as a FL, even in the presence of a magnetic field. We show that, due to this fact, the dynamics for the energy dissipation in the adiabatic regime is ruled by the IJL of Eq. (1) even beyond linear response. However, the mechanisms for the energy transport depend on the interactions and the spin polarization. We show that in systems without spin polarization (interacting and noninteracting), as well as in non-interacting systems (with and without spin-polarization), electrons with each spin orientation separately dissipate energy at a rate described by a Joule law $P_{\text {Joule }, \sigma}(t)=R_{0}\left[I_{C, \sigma}(t)\right]^{2}$. Instead, the interplay between many-body interactions and spin polarization leads to regimes where electrons with a given spin orientation exchange energy with electrons with the opposite spin orientation, although the total rate for the energy dissipation is described by Eq. (1).

The work is organized as follows. We present the theoretical treatment in Section II. In Section III we discuss the case where the quantum dot is non-interacting. We show that the exact description of the adiabatic dynamics is fully determined by the behavior of the charge susceptibility of the frozen system described by the equilibrium Hamiltonian frozen at a given time. The effect of many-body interactions is discussed in Section IV. In Section V we present numerical results obtained with Numerical Renormalization Group (NRG). For systems without spin polarization, we also use exact results of static properties obtained using the Bethe Ansatz (BA). We present the summary and conclusions in Section VI.

\section{THEORETICAL TREATMENT}

\section{A. Model}

We consider the system of Fig. 1 A driven quantum dot is connected to a normal lead of free electrons at zero temperature and chemical potential $\mu$. The full setup is described by an Anderson Hamiltonian,

$$
H(t)=H_{\text {dot }}(t)+H_{\text {res }}+H_{\mathrm{T}} .
$$

The first term describes the dot

$$
H_{\mathrm{dot}}(t)=\sum_{\sigma} \varepsilon_{d, \sigma}(t) n_{d \sigma}+U\left(n_{\uparrow}-\frac{1}{2}\right)\left(n_{\downarrow}-\frac{1}{2}\right),
$$

with $n_{d \sigma}$ denoting the number operator with spin $\sigma=\uparrow, \downarrow, U$ is the Coulomb repulsion, and $\varepsilon_{d, \sigma}(t)=\varepsilon_{0}+s_{\sigma} \frac{\delta_{Z}}{2}+\mathcal{V}_{g}(t)$ is the single-particle energy modulated by the applied gate voltage $V_{g}(t)$, with $\mathcal{V}_{g}(t)=e V_{g}(t)=V_{0} \sin (\Omega t), \delta_{Z}$ is the Zeeman splitting due to the presence of an external magnetic field, $s_{\sigma}= \pm 1$ for $\sigma=\uparrow, \downarrow$, and $-e$ is the charge of the electron. The reservoir is described by the Hamiltonian $H_{\text {res }}=\sum_{\sigma, k} \epsilon_{k} c_{k \sigma}^{\dagger} c_{k \sigma}$, which is assumed to have a constant density of states within a bandwidth $2 D$. The coupling between dot and reservoir is $H_{T}=V_{c} \sum_{k \sigma}\left[c_{k \sigma}^{\dagger} d_{\sigma}+h . c\right]$.

\section{B. Charge and energy adiabatic dynamics}

The conservation of the charge in the full system implies

$$
e \dot{n}_{d}(t)=e \sum_{\sigma} \dot{n}_{d \sigma}(t)=\sum_{\sigma} I_{C, \sigma}(t)
$$

where $n_{d \sigma}(t) \equiv\left\langle n_{d \sigma}(t)\right\rangle$ is the occupancy of the dot by electrons with spin $\sigma$ at time $t, I_{C, \sigma}(t)$ is the contribution of the electrons with spin $\sigma$ to the charge current flowing out of the dot at time $t$, and $e>0$ the elementary charge.

The power developed by the external ac source on the electron system is defined as ${ }^{20} P_{\text {ac }}(t)=-\langle\partial H / \partial t\rangle=-e \sum_{\sigma} n_{d \sigma}(t) \dot{V}_{g}(t)$. This leads to a net heat production in the electron system at a rate $\dot{Q}(t)=-P_{\text {ac }}(t) \stackrel{21}{2}$ We find it convenient to define the power

$$
P(t)=e \sum_{\sigma} n_{d \sigma}(t) \dot{V}_{g}(t)
$$

such that $P>0$ implies work delivered from the electron system against the ac sources. With this definition, the rate for the heat production in the electron system reads $\dot{Q}(t)=P(t)=$ $P_{\text {cons }}(t)+P_{\text {diss }}(t) \stackrel{22}{2}$ This power contains a purely ac component $P_{\text {cons }}(t)$ associated to the reversible heat produced by the conservative (Born-Oppenheimer) forces, and a dissipative component $P_{\text {diss }}(t)$ with a non-zero time average.

The dynamics of the heat production and the charge current is fully determined by $n_{d \sigma}(t)$. For low frequencies, the latter can be calculated within the adiabatic formalism of Ref. 24, 
which corresponds to linear-response in $\dot{V}_{g}(t)$ (see Appendix A). The result is

$$
n_{d \sigma}(t)=n_{f \sigma}(t)+e \Lambda_{\sigma}(t) \dot{V}_{g}(t)
$$

where $n_{f, \sigma}(t) \equiv\left\langle n_{d \sigma}\right\rangle_{t}$ is the snapshot occupancy of the dot, evaluated with the exact equilibrium density matrix $\rho_{t}$ corresponding to the Hamiltonian $H(t)$ frozen at the time $t$. The coefficient of the second term is

$$
\Lambda_{\sigma}(t)=-\lim _{\omega \rightarrow 0} \frac{\operatorname{Im}\left[\chi_{t}^{\sigma \sigma}(\omega)+\chi_{t}^{\sigma \bar{\sigma}}(\omega)\right]}{\hbar \omega},
$$

with $\bar{\uparrow}=\downarrow$ and $\bar{\downarrow}=\uparrow . \chi_{t}^{\sigma \sigma^{\prime}}(\omega)$ is the Fourier transform of the charge susceptibility $\chi_{t}^{\sigma \sigma^{\prime}}\left(t-t^{\prime}\right)=-i \theta\left(t-t^{\prime}\right)\left\langle\left[n_{d \sigma}(t), n_{d \sigma^{\prime}}\left(t^{\prime}\right)\right]\right\rangle_{t}$ evaluated with $\rho_{t}$.

In the case of the system with applied magnetic field, it is appropriate to analyze separately the current and the power developed by electrons with the different spin components. The current per spin can be calculated by the derivative of Eq. (6)

$$
I_{C, \sigma}(t)=e \frac{d n_{f, \sigma}}{d V_{g}} \dot{V}_{g}(t)+e^{2} \frac{d\left[\Lambda_{\sigma}(t) \dot{V}_{g}(t)\right]}{d t},
$$

where the first term is related to the static charge susceptibility through $d n_{f, \sigma} / d V_{g}=\chi_{t}^{\sigma \sigma}(0)$.

The frozen component $n_{f, \sigma}(t)$ contributes to the conservative component of this power, while the last term of Eq. (6) contributes to the non-conservative one. They read, respectively

$$
P_{\text {cons }, \sigma}(t)=e n_{f \sigma}(t) \dot{V}_{g}(t), \quad P_{\sigma}(t)=e^{2} \Lambda_{\sigma}(t)\left[\dot{V}_{g}(t)\right]^{2} .
$$

It is important to notice that the non-conservative components $P_{\sigma}(t)$ are not necessarily fully dissipative. They certainly contribute to the total dissipation, but they may also contain a non-dissipative "exchange" part $P_{\text {ex }}(t)$, such that $P_{\uparrow(\downarrow)}(t)=$ $\pm P_{\mathrm{ex}}(t)+P_{\mathrm{diss}, \uparrow(\downarrow)}(t)$. The exchange component is associated to time-dependent induced forces that are proportional to $\dot{V}_{g}(t)$. In this sense, these forces are akin to the "Lorentz" forces discussed in Ref. 25. However, in the present case they may develop work only instantaneously while the average over one period is zero.

The total power has conservative $P_{\text {cons }}(t)=\sum_{\sigma} P_{\text {cons }, \sigma}(t)$, and dissipative components $P_{\text {diss }}(t)=\sum_{\sigma} P_{\text {diss }, \sigma}(t)$, which read

$$
\begin{aligned}
& P_{\text {cons }}(t)=e \sum_{\sigma} n_{f \sigma}(t) \dot{V}_{g}(t), \\
& P_{\text {diss }}(t)=e^{2} \sum_{\sigma} \Lambda_{\sigma}(t)\left[\dot{V}_{g}(t)\right]^{2} .
\end{aligned}
$$

For later use we also define

$$
\begin{gathered}
\Lambda_{\sigma \sigma^{\prime}}(t)=-\lim _{\omega \rightarrow 0} \frac{\operatorname{Im}\left[\chi_{t}^{\sigma \sigma^{\prime}}(\omega)\right]}{\hbar \omega}, \\
P_{\sigma \sigma^{\prime}}(t)=e^{2} \Lambda_{\sigma \sigma^{\prime}}(t)\left[\dot{V}_{g}(t)\right]^{2} .
\end{gathered}
$$

When performing the averages over one period $\tau=2 \pi / \Omega$ for these two contributions to the power, $\bar{P}_{\text {cons, diss }}=$ $(1 / \tau) \int_{0}^{\tau} d t P_{\text {cons,diss }}(t)$, we find $\bar{P}_{\text {cons }}=0$ and $\bar{P}_{\text {diss }} \geq 0$ in accordance to the second law of thermodynamics.

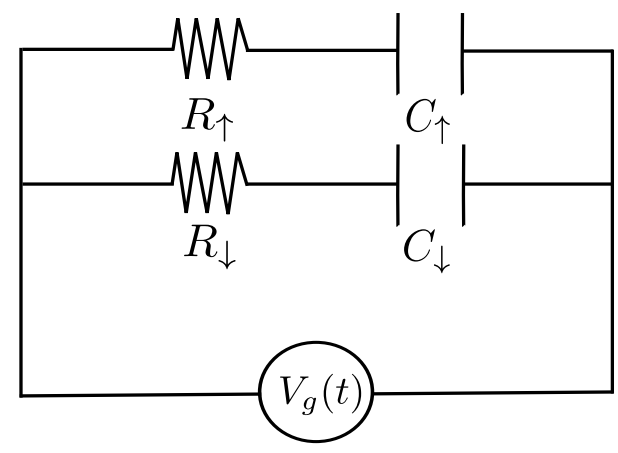

FIG. 2. (Color online) Sketch of the circuit. Upper and lower branch corresponds to $\uparrow$ and $\downarrow$ spin channels.

We see that the full charge and energy dynamics in the adiabatic regime is completely determined by the behavior of the frozen charge susceptibility $\chi_{t}^{\sigma \sigma^{\prime}}(\omega)$, irrespective of the strength of the interactions and the amplitude of the driving potential.

\section{Analogy to the non linear classical circuit}

We now discuss the representation of the equations for the dynamics of the charge and energy introduced in the previous section, in terms of a classical non-linear circuit. We find it convenient to treat the two spin channels separately as a circuit with two branches (one for each spin species) connected in parallel to the ac source, as sketched in Fig. 2. Each branch contains a capacitance $C_{\sigma}(t)$ in series with a resistance $R_{\sigma}(t)$. We assume that the equation relating the current through each branch of the circuit with the potential $V_{g}(t)$ is

$$
I_{C, \sigma}(t)=-C_{\sigma}(t) \dot{V}_{g}(t)+e^{2} \frac{d\left[R_{\sigma}(t) C_{\sigma}(t)^{2} \dot{V}_{g}(t)\right]}{d t} .
$$

As discussed in Appendix B this equation corresponds to a true macroscopic classical $\mathrm{RC}$ circuit in the non-linear lowfrequency regime satisfying $\Omega R_{\sigma} I_{\sigma} \ll 1$. Identifying linear and quadratic terms in $\dot{V}_{g}$ in the above equation with those of the quantum current Eq. (8) one obtains

$$
C_{\sigma}(t)=-e \frac{d n_{f, \sigma}(t)}{d V_{g}}=-e \chi_{t}^{\sigma \sigma}(0), \quad R_{\sigma}(t) C_{\sigma}^{2}(t)=e^{2} \Lambda_{\sigma}(t)
$$

Here, unlike the linear case, the non-linear capacitance $C_{\sigma}(t)$ and resistance $R_{\sigma}(t)$ are, in general, functions of $t$. In terms of these coefficients, the dissipated power 10 reads

$$
P_{\mathrm{diss}}(t)=\sum_{\sigma} R_{\sigma}(t) C_{\sigma}^{2}(t)\left[\dot{V}_{g}(t)\right]^{2} .
$$

We see that for the case where $R_{\sigma}(t)=R_{0}$, Eq. (14) reduces to the IJL described by Eq. (1), which is immediately derived by retaining only the first term of Eq. (8). In fact, the latter is the only term of Eq. (8) which has a contribution $\propto\left[\dot{V}_{g}(t)\right]^{2}$ to $P_{\text {diss }}(t)$, since $d \Lambda_{\sigma}(t) / d t=\left(d \Lambda_{\sigma}(t) / d V_{g}(t)\right) \dot{V}_{g}(t)$. 
The above equations are exact and valid in general within the adiabatic regime. However, in order to establish a meaningful correspondence between the charge and energy dynamics of the quantum system and the classical circuit of Fig. 2, the coefficients defined in Eq. (13) should also verify $R_{\sigma}(t)>0$ and $C_{\sigma}(t)>0$. As we will discuss in detail in the next sections, such a correspondence is valid in the system without spin polarization $\left(\delta_{Z}=0\right)$ for arbitrary Coulomb interaction $U$, as well as in the non-interacting case $(U=0)$ with arbitrary $\delta_{Z}$. We also argue in Sections IV and V that the dynamics of the driven interacting quantum dot in the presence of a magnetic field cannot be interpreted in terms of the circuit of Fig. 2.

\section{NON-INTERACTING ELECTRONS}

\section{A. Review of the spinless case}

The expressions of the previous sections are completely general and valid for arbitrary temperatures, for non-interacting as well as interacting systems. We now relate them to the non-interacting results for spinless electrons of Refs. 12 , 19, 21, and 24. This corresponds to the Hamiltonian (3) with $U=\delta_{Z}=0$ and with only one spin species. Following Refs. 19, 21, and 27, we get

$$
\begin{aligned}
& C(t)=-e \int d \epsilon \frac{\partial f}{\partial \epsilon} \rho_{f}(t, \epsilon), \\
& \Lambda(t)=-\frac{h}{2} \int d \epsilon \frac{\partial f}{\partial \epsilon}\left[\rho_{f}(t, \epsilon)\right]^{2},
\end{aligned}
$$

where $\rho_{f}(t, \epsilon)=(\Delta / \pi) /\left[\left(\epsilon-\varepsilon_{d}(t)\right)^{2}+\Delta^{2}\right]$ is the noninteracting frozen density of states of the quantum dot connected to a reservoir with constant density of states $v, \Delta=$ $\pi \nu V_{c}^{2}$, and $f(\epsilon)$ is the Fermi distribution function.

The resistance can be directly calculated from Eq. (13). At $T=0$, we have $-\partial f / \partial \epsilon=\delta(\epsilon-\mu)$. Hence, the coefficients simplify to $C(t)=e^{2} \rho_{f}(t, \mu)$ and $R(t)=R_{0}=h /\left(2 e^{2}\right)$. The latter corresponds to the universal resistance quantum for a single channel. By substituting these expressions in $P_{\text {diss }}(t)$ and $I_{C}(t)$, and keeping terms up to $O\left(\dot{V}_{g}^{2}\right)$, we recover the IJL of Eq. (1) as in Ref.19. Interestingly, we get the same expression of the current $I_{C}(t)$ in the non-interacting limit as the one of Ref. 12. However the definition of $R(t)$ presented there differs from the definition of Eq. (13) with $\Lambda(t)$ given by Eq. (15). Such difference should be traced back to the equation for the non-linear circuit (12). Unlike the one considered in Ref. 12 , Eq. (12) includes the factor $R C$ inside the time-derivative of the second term. The structure of the latter Eq. is motivated by the adiabatic expansion of the occupancy Eq. (6), by identifying the coefficient $\Lambda(t)$ as the dissipative contribution. Remarkably, our definition of $R(t)$ can be easily related to $R_{0}$ in the limit of $T=0$ and it consistently leads to the Joule law of Eq. (1), while it is also in agreement with the effective resistance defining the noise ${ }^{12}$

\section{B. Spinfull electrons}

We now consider the case with $U=0$ and arbitrary $\delta_{Z}$. Notice that for non-interacting electrons the "crossed-susceptibility"

$\chi_{t}^{\sigma, \bar{\sigma}}(\omega)=0$. Hence the coefficient $\Lambda_{\sigma}(t)$ is fully determined by the susceptibilities $\chi_{t}^{\sigma, \sigma}(\omega)$. The calculations of Refs. 12, 19, 21, and 24 can be easily extended to non-interacting electrons with spin.

The frozen occupancy of the quantum dot with spin $\sigma$ is

$$
n_{f, \sigma}(t)=\int d \epsilon \rho_{f, \sigma}(t, \epsilon) f(\epsilon),
$$

where $\rho_{f, \sigma}(t, \epsilon)=\left(\Delta_{\sigma} / \pi\right) /\left[\left(\epsilon-\varepsilon_{d, \sigma}(t)\right)^{2}+\Delta_{\sigma}^{2}\right]$. For this model $\partial \rho_{f, \sigma}(t, \epsilon) / \partial t=e\left(\partial \rho_{f, \sigma}(t, \epsilon) / \partial \epsilon\right) \dot{V}_{g}(t)$. Hence, after integrating by parts the above equation, we get for $T=0$

$$
C_{\sigma}(t)=e \rho_{f, \sigma}(t, \mu)
$$

In addition, we get an expression like 15 for each spin orientation $\sigma$. For $T=0$, it reads

$$
\Lambda_{\sigma}(t)=\frac{h}{2}\left[\rho_{f, \sigma}(t, \mu)\right]^{2}=\frac{h}{2}\left[\chi_{t}^{\sigma \sigma}(0)\right]^{2},
$$

which is a special case of the Korringa-Shiba (KS) law discussed in the next section. Inserting these expressions in Eqs. (13) we obtain $R_{\sigma}(t)=R_{0}$. Substituting in 100 , we see that the dissipated power is ruled by the IJL of Eq. (1).

Therefore, for non-interacting electrons, Korringa-Shiba law of Eq. (18) implies that there is a full one-to one correspondence between the charge and energy dynamics of the driven electron system and the two-branch circuit sketched in Fig. 2 with resistances $R_{\sigma}(t)=R_{0}$, even when the electrons are spinpolarized. This also means that the ac forces associated to the induced charge for each spin orientation dissipate heat in the form of a Joule law, $P_{\sigma}(t)=R_{0}\left[I_{C, \sigma}(t)\right]^{2}=P_{\text {Joule }, \sigma}(t)$. Hence, $P_{\text {diss }}(t)=\sum_{\sigma} P_{\text {Joule }, \sigma}(t)=R_{q}\left[I_{C}(t)\right]^{2}$, with $I_{C}(t)=\sum_{\sigma} I_{C, \sigma}(t)$ and $R_{q}=R_{0} / 2$.

\section{INTERACTING ELECTRONS}

\section{A. Exact results}

For interacting electrons, the crossed susceptibility $\chi_{t}^{\sigma, \bar{\sigma}}(\omega)$ contributes to the coefficient $\Lambda_{\sigma}(t)$, in addition to $\chi_{t}^{\sigma, \sigma}(\omega)$. For Fermi liquids, an important relation exists for the total charge susceptibility $\chi_{t}^{c}(\omega)=\sum_{\sigma, \sigma^{\prime}} \chi_{t}^{\sigma, \sigma^{\prime}}(\omega)$, which receives the name of Korringa-Shiba law. ${ }^{26}$ In the non-interacting case, it is expressed in Eq. (18). In the interacting case, it is a nontrivial result, which was originally proved by Shiba in the Anderson model $^{26}$ and later generalized by Fillipone et al. when a magnetic field is also considered. ${ }^{10,11}$ It reads

$$
\lim _{\omega \rightarrow 0} \frac{\operatorname{Im}\left[\chi_{t}^{c}(\omega)\right]}{\hbar \omega}=-\frac{h}{2} \sum_{\sigma}\left[\chi_{t}^{\sigma \sigma}(0)\right]^{2} .
$$

This relation has been used to study the present problem within the linear response regime ${ }^{7-11}$ Here, we show that the 
Korringa-Shiba law Eq. 19 is equivalent to the instantaneous Joule law Eq. (1), even in the presence of a magnetic field and also in the non-linear response regime.

In fact, from Eqs. (7) and (10) and taking into account that Eq. (19) is satisfied, we have

$$
P_{\text {diss }}(t)=\frac{e^{2} h}{2} \sum_{\sigma}\left[\chi_{t}^{\sigma \sigma}(0)\right]^{2} \dot{V}_{g}(t)^{2}
$$

On the other hand, the -up to $O\left(\dot{V}_{g}(t)\right)$ - charge current with spin $\sigma$ is given by the first term of Eq. (8) and reads

$$
I_{C, \sigma}(t) \simeq e \frac{\partial n_{f \sigma}(t)}{\partial V_{g}(t)} \dot{V}_{g}(t)=e \chi_{t}^{\sigma \sigma}(0) \dot{V}_{g}(t) .
$$

Then, substituting in Eq. (20), we get

$$
P_{\text {diss }}(t)=\frac{h}{2 e^{2}} \sum_{\sigma}\left[I_{C, \sigma}(t)\right]^{2},
$$

which is, precisely, the IJL. This result holds for electrons with and without spin polarization, in the non-linear as well as in the linear regimes.

\section{B. Non-polarized electrons and the representation by the classical circuit}

In the case of non-polarized electrons, where the two spin orientations are equivalent. The total charge current $I_{C}(t)=$ $\sum_{\sigma} I_{C, \sigma}(t)$ associated to the change in the dot occupancy by up and down spins is given by [see Eqs. (8) and (13)]

$$
I_{C}(t)=-C(t) \dot{V}_{g}(t)+e^{2} \frac{d\left[\Lambda^{c}(t) \dot{V}_{g}(t)\right]}{d t},
$$

with $C(t)=\sum_{\sigma} C_{\sigma}(t)$, and $\Lambda^{c}(t)=\sum_{\sigma} \Lambda_{\sigma}(t)$.

The definition of the resistance given in Eq. (13), along with $C(t)=-e^{2} \chi_{t}^{c}(0)$ and the KS relation Eq. [19], lead to the resistance $R_{0}$ for each spin channel or an equivalent resistance $R_{q}=R_{0} / 2$ for the equivalent circuit of Fig. 1 . In fact, because of the equivalence of both branches of the circuit of Fig. 2, the voltage drop at the middle point between the resistance and capacitance for each branch are the same. Therefore, one can connect this two points with a cable carrying no current and the circuit becomes equivalent to that at the top of Fig. 1, with an effective resistance $R_{q}^{-1}=2 R_{0}^{-1}$ in series with the effective capacitance $C(t)=2 C_{\sigma}(t)$. In addition, the arguments of the next section as well as the numerical results will show that $C(t) \geq 0$. Hence, for non-polarized electrons, the behavior of the charge and energy dynamics is consistent with the representation of the setup in terms of the parallel circuit of Fig. 2 or the equivalent one of Fig. 1. The charge dynamics is described by Eq. (23) with $e^{2} \Lambda^{c}(t)=R_{q}[C(t)]^{2}$. while the dissipated power obeys the IJL Eq. (22).

\section{Polarized electrons in the random-phase approximation}

In the case of polarized electrons, the two spin orientations are not equivalent and it is not easy to analyze the dynamics by simple analogy to the classical circuit. It is important to notice that the Coulomb interaction effectively renormalizes the gate voltage at a given time. At the mean field level, this can be accounted by an occupancy-dependent term $U\left\langle n_{f, \bar{\sigma}}\right\rangle$ which adds to $\mathcal{V}_{g}(t)$ in the effective local energy experienced by an electron with spin $\sigma$ at the quantum dot. Here, we will analyze the consequence of this effect on the basis of the behavior of the charge susceptibility in the "random phase approximation" (RPA). In the next section we will present a more accurate analysis based on NRG results.

RPA corresponds to calculating the dynamic susceptibility from the summation of an infinite perturbative series of "bubble" diagrams. The result in the present case is

$$
\chi_{t}^{\sigma \sigma}(\omega)=\frac{\chi_{t}^{0 \sigma}(\omega)\left[1+U \chi_{t}^{0 \bar{\sigma}}(\omega)\right]}{1-U^{2} \chi_{t}^{0 \sigma}(\omega) \chi_{t}^{0 \bar{\sigma}}(\omega)},
$$

where $\chi_{t}^{0 \sigma}(\omega)$ is the susceptibility for $U=0$. The latter is a function of the gate voltage $V_{g}(t)$ and satisfies the KS relation (18). The static limit is given by Eq. (17), $\chi_{t}^{0 \sigma}(0)=$ $-\rho_{f, \sigma}(\mu)$. In the case of non-polarized electrons, where the two spin orientations are equivalent, we have $\chi_{t}^{\sigma \sigma}(\omega)=$ $\chi_{t}^{0 \sigma}(\omega) /\left[1+U \chi_{t}^{0 \sigma}(\omega)\right]$. Hence, the calculation of the capacitance gives $C_{\sigma}(t)=C_{\sigma, 0}(t) /\left[1+U C_{\sigma, 0}(t) / e\right]$, where $C_{\sigma, 0}(t)$ is the capacitance of the non-interacting system (17), which indicates that $C_{\sigma}(t) \geq 0$.

In the case of polarized electrons we have situations where $\left|\chi_{t}^{0 \sigma}(0)\right| \ll\left|\chi_{t}^{0 \bar{\sigma}}(0)\right|$ or vice versa, in which case Eq. (24) leads to negative values of the coefficient $C_{\sigma}(t)$ for large enough $U$. This corresponds to a current between the reservoir and the dot which opposes to the sense of circulation imposed by the voltage drop. Furthermore, after some algebra from (24) and the KS relation for the non-interacting susceptibilities (18), we can see that in such situations the signs of $\Lambda_{\sigma}(t)$ and $\Lambda_{\bar{\sigma}}(t)$ are opposite. This would correspond to instantaneous exchange of power between the two spin species, $P_{\sigma}(t) \sim P_{\mathrm{ex}}(t) \sim-P_{\bar{\sigma}}(t)$. We can interpret this behavior as electrons with spin $\sigma$ receiving energy from the electrons with spin $\bar{\sigma}$ to move against the external voltage drop. In addition to this component, there is a dissipative component of the power satisfying the IJL [see Eq. [22)]. Since the behavior explained above is not expected in a capacitive circuit element, we conclude that the representation of the dynamics of the driven interacting quantum dot in the presence of a magnetic field is not properly represented by a circuit like that of Fig. 2. In the next section, we will verify that such a behavior indeed takes place when the susceptibilities are exactly calculated with NRG.

\section{NUMERICAL RESULTS FOR THE NON-LINEAR INTERACTING REGIME}

We now turn to further analyze the adiabatic fully interacting case for arbitrary amplitudes of the driving on the basis of numerical results. We use the numerical renormalization group (NRG) algorithm of Ref. 30 to compute the frozen occupancy of the dot $n_{f \sigma}(t)$ and the charge susceptibility $\chi_{t}^{\sigma}(\omega)$. We stress that the evaluation of these two quantities corresponds to an 

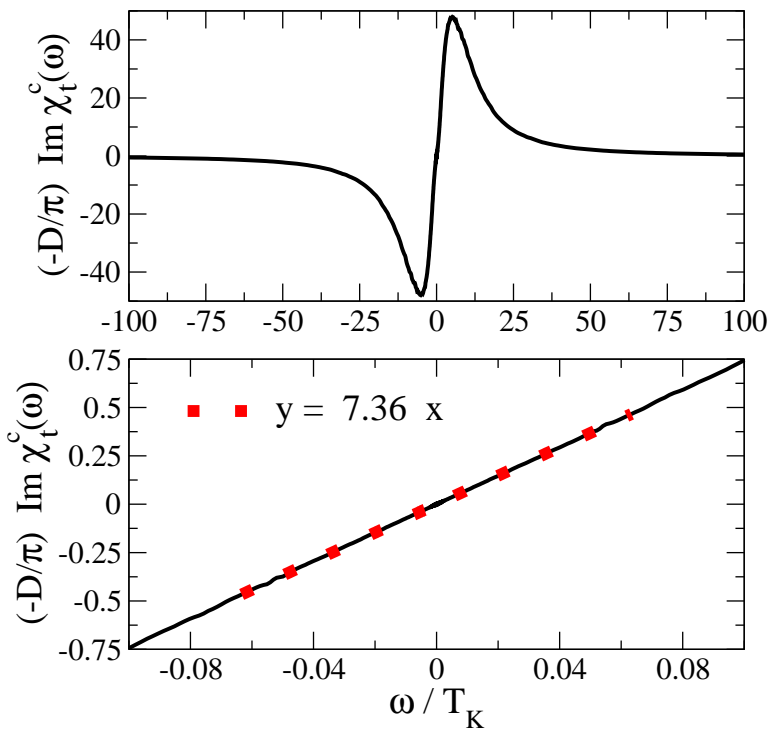

FIG. 3. (Color online) Imaginary part of the dynamic susceptibility as a function of frequency for $\Omega t=\pi / 2, \Delta=8 \times 10^{-4} D, \varepsilon_{0}=\mu=0$, $U=0.05 D, V_{0}=0.024 D$ and $T=0$.

equilibrium calculation with the Hamiltonian $H(t)$ frozen at the time $t$ (for details see Appendix C). We also use analytical expressions of the impurity occupancy obtained from Bethe ansatz $(B A)^{28}$ using the procedure outlined in the appendix of Ref. 29.

\section{A. Results for zero magnetic field}

In a system without spin polarization, the two spin orientations are equivalent and the dynamics of the quantum system is fully equivalent to that of the circuit of Fig. 2 or that with the equivalent components sketched in Fig.1. As argued in the previous section, the two resistances can be simply substituted by the resistance $R_{q}=R_{0} / 2$ in series with the capacitance $C(t)=2 C_{\uparrow}(t)=2 C_{\downarrow}(t)$. The total current circulating through the single branch of the equivalent circuit is given by Eq. (23). The total dissipated power is given by Eq. (10) with $\Lambda^{c}(t)=\sum_{\sigma} \Lambda_{\sigma}(t)$.

\section{Benchmark}

We start with a benchmark of the numerical results calculated with NRG against exact analytical results calculated with BA. In particular, we verify that the Korringa-Shiba law Eq. (19) is satisfied. To this end, we analyze the Fourier transform of the total charge susceptibility $\chi_{t}^{c}\left(t-t^{\prime}\right)=-i \theta(t-$ $\left.t^{\prime}\right) \sum_{\sigma \sigma^{\prime}}\left\langle\left[n_{d \sigma}(t), n_{d \sigma^{\prime}}\left(t^{\prime}\right)\right]\right\rangle_{t}$ evaluated with the exact equilibrium density matrix $\rho_{t}$. An example is shown in Fig. 3 for a fixed time. For the parameters of the figure we estimate a Kondo temperature $T_{K} \approx 2.94 \times 10^{-4} D$, where $D=1$ is half the band width used in the NRG calculations. The general aspect of the curve is similar to that reported previously $\stackrel{\underline{8}}{\mathrm{Ac}} \mathrm{Ac}$
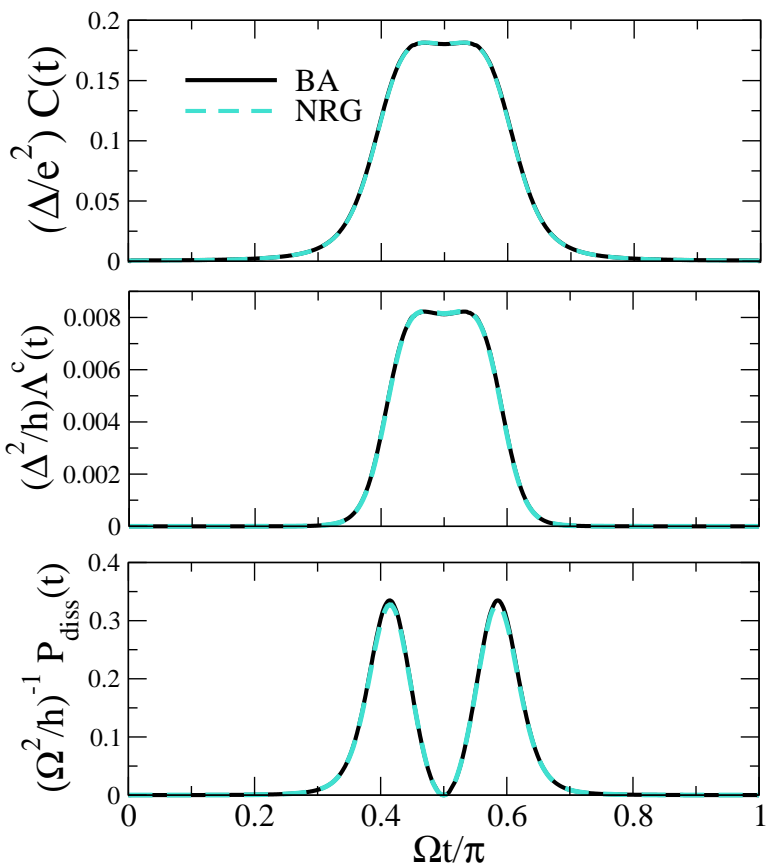

FIG. 4. (Color online) (a) capacitance $C(t)$ (b) Dissipation coefficient $\Lambda^{c}(t)$, and (c) dissipated power $P_{\text {diss }}(t)$ in the interacting non-linear regime, as a function of time, calculated with two techniques. Other parameters as in Fig. 3

cording to the Korringa-Shiba law Eq. (19) one has for $\omega \rightarrow 0$

$$
\operatorname{Im} \chi_{t}^{c}(\omega)=-\omega \frac{h}{4}\left[\chi_{t}^{c}(0)\right]^{2}=-\omega \frac{h}{2} \sum_{\sigma}\left[\chi_{t}^{\sigma}(0)\right]^{2} .
$$

Fitting the results for $\omega \ll T_{K}$ as shown in the bottom panel of Fig. 3 we obtain $-\operatorname{Im} \chi_{t}^{c}(\omega) / \pi=7.36 \hbar^{2} \omega /\left(D T_{K}\right)$, or $-\operatorname{Im} \chi_{t}^{c}(\omega)=\Lambda^{c} \hbar \omega$, with $\Lambda^{c}=7.36 h /\left(2 T_{K} D\right)=12520 h / D^{2}$. Calculating the charge susceptibility by numerical differentiation of the total occupancy $n_{f}(t)=\sum_{\sigma} n_{f \sigma}$, obtained with NRG from

$$
\chi_{t}^{c}(0)=\sum_{\sigma} \frac{d n_{f \sigma}(t)}{d \varepsilon_{d}}
$$

with $\varepsilon_{d} \equiv \varepsilon_{d, \uparrow}(t)=\varepsilon_{d, \downarrow}(t)$ and using Eq. 25), we obtain $\Lambda^{c}=12732 h / D^{2}$, a value $1.7 \%$ larger. By numerical differentiation of the BA occupancy we obtain $\Lambda^{c}=12690 h / D^{2}$, which differs from the previous result by $0.3 \%$. This deviation might be due to the fact that in the BA procedure we take $D \rightarrow \infty$.

We have also checked the Fermi liquid relation Eq. 25) for other values of the parameters obtaining agreement with the static results within about $2 \%$. This confirms the validity of these relations. The slight discrepancy between both NRG results is likely to be due larger numerical errors in the dynamic calculation.

In Fig. 4 4 we represent the static susceptibility, proportional to the total capacitance $C(t)=-e^{2} \chi_{t}^{c}(0)$ in the non-linear circuit analog, $\Lambda^{c}(t)$ and the total dissipated power $P_{\text {diss }}(t)$ as a function of time. The total resistance is for all times $R_{q}=R_{0} / 2$. 


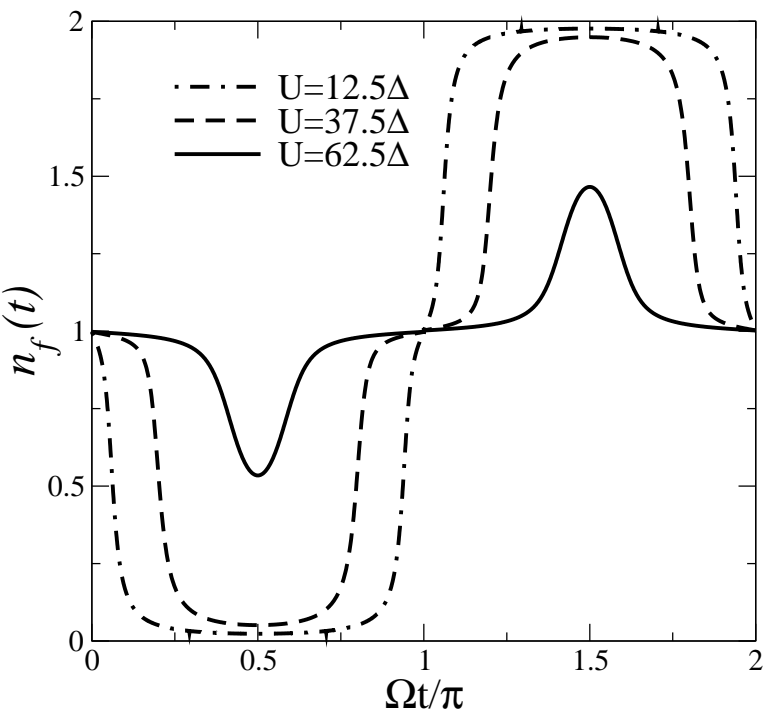

FIG. 5. (Color online) occupancy of the quantum dot as a function of time for different values of the Coulomb interaction (indicated in the Fig). Other parameters as in Fig. 3

The other parameters are the same as in the previous case, which was limited to $t=\pi /(2 \Omega)$. One sees that the NRG and BA results agree very well for all times.

\section{Charge and energy dynamics}

From the practical point of view, it is easier to calculate static properties than dynamic ones. In addition, static quantities can be calculated exactly with BA. Hence, in what follows we calculate $n_{f, \sigma}(t)$. Then, we calculate the static susceptibility from Eq. (26). Finally, we use the Korringa-Shiba relation Eq. 25] to derive the dynamic response function $\Lambda^{c}(t)$.

Results for the total frozen occupancy of the dot as a function of time, $n_{f}(t)$, are shown in Fig. 5 These correspond to a given driving amplitude and frequency, and different values of the Coulomb repulsion. To analyze these results, let us start by focusing on the plot with dashed-dot lines, corresponding to the smallest $U$. At $t=0$ the dot is at the half-filling configuration, corresponding to a mean charge $n_{f}(0)=1$. As a function of $t, V_{g}$ increases and the occupancy of the dot decreases. In particular, when $\varepsilon_{d}(t) \sim U / 2$, the quantum dot becomes empty and remains in that configuration while $V_{g}$ passes through its maximum at $\Omega t=\pi / 2$. As the time continues to increase, the dot begins to get filled again with one electron. For larger time, $V_{g}$ continues to decrease and the single-particle energy of the dot $\varepsilon_{d}(t)$ reaches the value $\sim-U / 2$. The dot gets filled with two electrons and remains at this occupancy as $V_{g}$ passes through its minimum at $\Omega t=3 \pi / 2$. Finally, the occupancy decreases to reach the half-filled configuration, as the gate voltage completes the period. For the larger values of $U$ shown in the figure, we can observe similar features. For the largest one, corresponding to the solid lines, the dot does not reach the occupancy with zero and two electrons, since for all times $\left|\varepsilon_{d}(t)\right|<U / 2$.
The changes in the occupancy of the dot as a function of time, generate a charge current between the dot and the reservoir, according to Eq. 23). The corresponding capacitance, $C(t)$, the dissipation coefficient $\Lambda^{c}(t)$ and the dissipated power $P_{\text {diss }}(t)$ are shown in Fig. 6 for the same values of $U$ shown in Fig. 5 We can identify features in the capacitance associated to the static charge susceptibility (26). Due to the Korringa-Shiba law, similar features are also found in the dynamic coefficient $\Lambda^{c}(t)$. This determines also the behavior of the dissipated power shown in the lowest panel of the figure, where we can distinguish the peaks corresponding to the IJL described by Eq. (1).

To close this section, we comment on the relation between the features characterizing the charge and energy dynamics and the behavior of the local frozen density of states at the quantum dot as a function of time. For gate voltages close to the symmetric configuration satisfying $\left|\varepsilon_{d}(t)\right| \sim 0$ there is one electron in the quantum dot and the density of states has typically a resonant peak at the Fermi energy (the Kondo resonance) and charge-transfer (or Coulomb-blockade) peaks at high energies $\sim \pm U / 2{ }^{23}$ As the gate voltage moves away from the symmetric configuration within the range $\left|\varepsilon_{d}(t)\right| \leq U / 2$ the dot remains filled with a single electron, the Kondo resonance persists at the Fermi energy while the high-energy peaks move rigidly following $\varepsilon_{d}(t)$ (details of the evolution of the spectral weight can be found in e.g. Ref. 31). When $\varepsilon_{d}(t) \sim \pm U / 2$, one of these peaks becomes aligned with the Fermi energy of the reservoir and the dot changes its occupancy to 0 or 2 electrons for $\varepsilon_{d}(t)= \pm U / 2$, respectively. At the time this happens, a current flows towards or from the reservoir, respectively. This exchange of charge between the dot and the reservoir is accompanied by an instantaneous dissipation of energy in the form of a Joule law, as described by Eq. (11). This is reflected in the peaks of $C(t), \Lambda^{c}(t)$ and $P_{\text {diss }}(t)$ shown in Fig. 6 ,

In all the processes discussed, the Kondo resonance does not play any significant role. Therefore, the behavior of Figs. (5) and (6) is also representative of the Coulomb blockade regime taking place at finite temperatures when the coupling to the reservoir is very weak. Interestingly, this behavior is also similar to what is observed in experiments of the compressibility of strongly correlated quantum dots, which are also related to the behavior of the charge susceptibility at the Fermi energy 32

\section{B. Results for finite magnetic field}

The behavior of the time-dependent occupancies for $\uparrow$ and $\downarrow$ spins in the presence of a Zeeman splitting $\delta_{Z}$ at the quantum dot are shown in Fig. 7 for different values of $U$. The upper, middle and bottom panels correspond, respectively to the same parameters as the plot in dot-dashed, dashed and solid lines of Fig. 5, with the additional ingredient of a magnetic field. In all the cases, the dot is in the half-filled configuration at $t=0\left(n_{f}(0)=1\right)$ and is predominantly occupied by electrons with spins parallel to the direction of the magnetic field (in this case $\downarrow$ ). The magnitude of the Zeeman splitting is chosen larger than the Kondo temperature $T_{K}$ for all times. Hence, for one electron in the dot, the system is in the local 

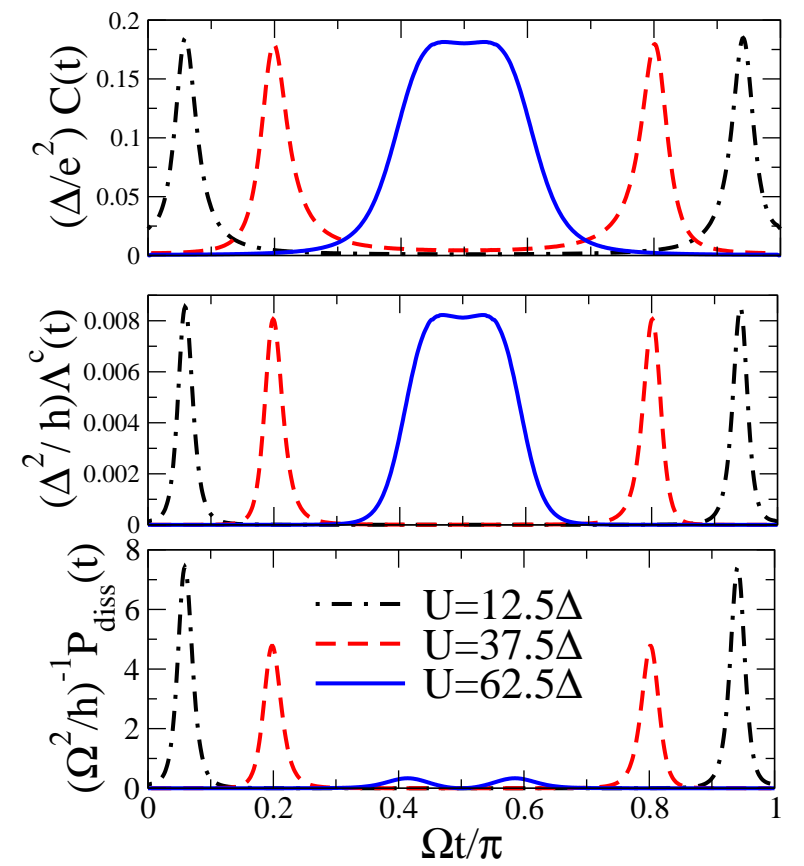

FIG. 6. (Color online) Same as Fig. 4 4 calculated with BA for several values of $U$.

moment regime of the Anderson model with a significant spin polarization.

Starting from the symmetric configuration at $t=0$, the energy of the localized electrons for both spin orientations $\varepsilon_{d, \sigma}(t)$ increases in time and the occupancy of the quantum dot evolves to the empty configuration when $\varepsilon_{d, \downarrow}(t) \sim U / 2$ (this situation is however not reached for the largest $U$ considered). As this happens, of course also the local spin of the quantum dot vanishes. After passing through its maximum at $\Omega t=\pi / 2, \varepsilon_{d, \sigma}(t)$ decreases and the quantum dot becomes again filled with a $\downarrow$ electron when $\varepsilon_{d, \downarrow}(t) \sim U / 2$. As $\varepsilon_{d, \sigma}(t)$ continues to decrease towards its minimum at $\Omega t=3 \pi / 2$, and additional electron occupies the quantum dot when $\varepsilon_{d, \downarrow}(t) \sim-U / 2$. This implies again a vanishing total spin at the quantum dot and as a consequence, the magnetic field does not lead to a spin polarization. In fact, we see in all the panels of the figure that the two occupancies differ only for values of the gate voltage where the total mean occupancy is close to one electron, in which case, there is a finite spin polarization at the quantum $\operatorname{dot}, \sum_{\sigma} n_{f, \sigma}(t) \neq 0,2$.

We note that for $\varepsilon_{0}=\mu=0$ and any magnetic field the Hamiltonian is invariant under the following transformation: $t \rightarrow-t$ and

$$
d_{\uparrow}^{\dagger} \rightarrow d_{\downarrow}, d_{\downarrow}^{\dagger} \rightarrow-d_{\uparrow}, \quad c_{k \uparrow}^{\dagger} \rightarrow-c_{k^{\prime} \downarrow}, c_{k \downarrow}^{\dagger} \rightarrow c_{k^{\prime} \uparrow},
$$

assuming a symmetric conduction band such that for any eigenstate $k$ of the isolated band, there is another one $k^{\prime}$ with $\epsilon_{k^{\prime}}=-\epsilon_{k}$. As a consequence of this symmetry, $n_{f, \uparrow}(t)=$ $1-n_{f, \downarrow}(-t)$ as can be seen in the figure.

Focusing on the interval $0<\Omega t<\pi / 2$ of Fig. 7 for which the one-site energy of the dot increases, we see that the expected decrease in the occupancy for the majority down spin
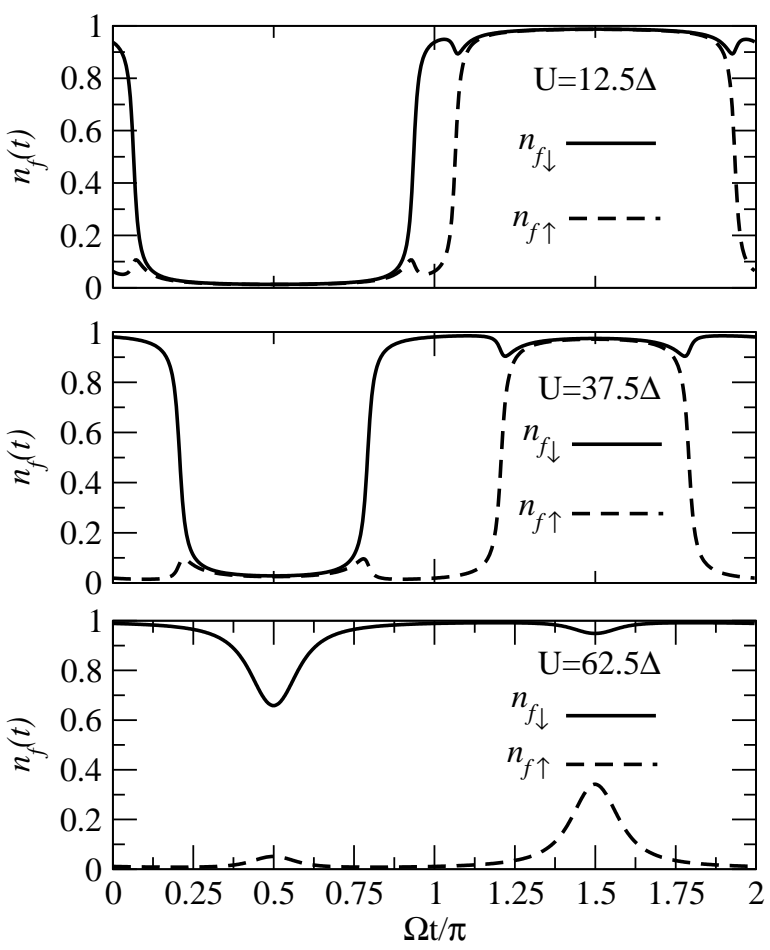

FIG. 7. (Color online) Frozen occupancies $n_{f, \uparrow}(t)$ and $n_{f, \downarrow}(t)$ for a Zeeman splitting $\delta_{Z}=10^{-3} \mathrm{D}$ and different values of $U$. Other parameters are the same as in the previous Figs.

is accompanied by an increase in the occupancy of the minority up spin, denoting a charge susceptibility of opposite sign for spin up. As already mentioned in Section IV. C, within a mean field description, the effective local energy for spin up is $\varepsilon_{d, \uparrow}(t)=\varepsilon_{0}+\frac{\delta_{Z}}{2}+\mathcal{V}_{g}(t)+U n_{f \downarrow}$, and the increase in $\mathcal{V}_{g}(t)$ is overcome by the decrease in $U n_{f \downarrow}$ for large enough $U$ and $\left|\chi_{t}^{\downarrow \downarrow}\right|$. For other parts of the cycle similar arguments can be followed, in particular using the symmetry transformation Eq. (27).

The behavior of the coefficients $\Lambda_{\sigma}$, which determine the nonconservative component of the power, is illustrated in Fig. 8 for the largest value of $U$ shown in Fig. 7 These coefficients display a very interesting behavior as functions of time. Both exhibit features at those times where the occupancy of the quantum dot experiences a significant fluctuation, implying a finite charge current flowing between the reservoir and the quantum dot. The coefficient $\Lambda_{\downarrow}(t)$, associated to electrons with the majority spin polarization is always positive in the interval of time shown. Note that the symmetry transformation Eq. 27) implies that $\Lambda_{\uparrow}(t)=\Lambda_{\downarrow}(-t)$. Instead, the coefficient $\Lambda_{\uparrow}(t)$, which is related to the minority spin orientation can be negative. Notice that this is in strong contrast to the nonpolarized case, where the two coefficients are identical and positive. The coefficients $\Lambda_{\sigma}(t)$ do not separately satisfy the Korringa-Shiba law of the non-interacting system expressed by Eq. (18). This can be seen by comparing the plots in symbols with those in lines in the upper panel of the figure. However, the total coefficient $\Lambda^{c}(t)$ obeys the Korringa-Shiba 

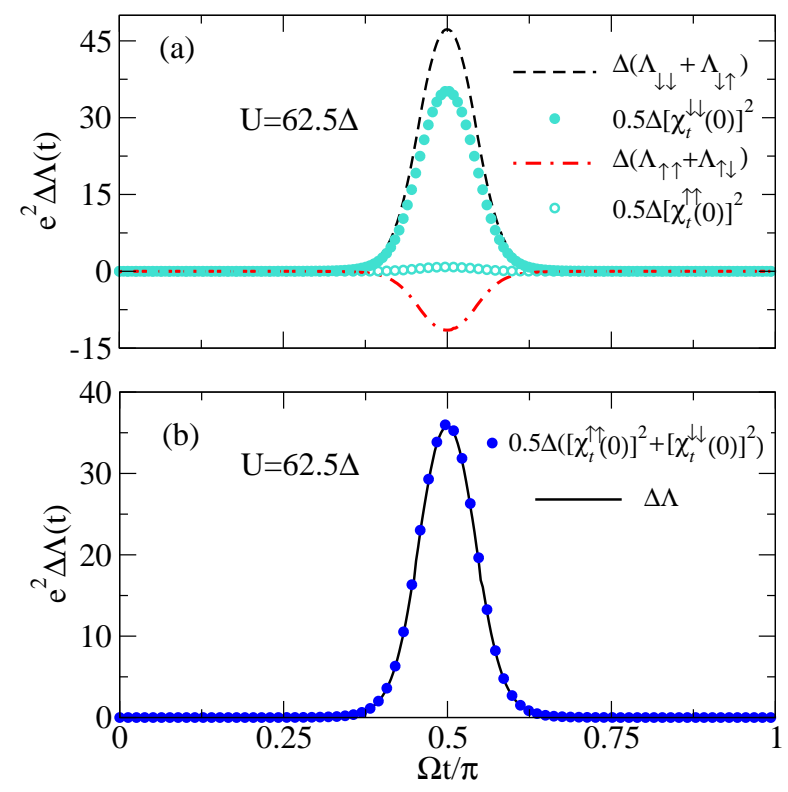

FIG. 8. (Color online) Analysis of the Korringa-Shiba laws of Eq. (18) and (19). The functions $\Lambda^{\uparrow}(t)$ and $\Lambda^{\downarrow}(t)$ are compared with $\left[\chi_{t}^{\uparrow \uparrow}(0)\right]^{2}$ and $\left[\chi_{t}^{\downarrow \downarrow}(0)\right]^{2}$ for $U=0.05 D$. Other parameters are the same as in Fig. 7

relation [19), as shown in the bottom panel of the figure.

The corresponding behavior of the developed power is presented in Fig. 9 for the smallest and largest value of $U$ considered in Fig. 7 We show in the upper panels the coefficients $\Lambda_{\sigma}(t)$, and in the middle panels the corresponding nonconservative powers $P_{\sigma}(t)$. Note that $P_{\uparrow}(t)=-P_{\downarrow}(-t)$ as a consequence of the symmetry transformation Eq. 27]. For comparison, the lower panels show the IJL per spin, $P_{\mathrm{Joule}, \sigma}(t)$. All these quantities have features at those times $t$ where the occupancy of the dot changes and a charge current is established between the dot and the reservoir.

As mentioned in the discussion of the previous figure, the striking feature is the different sign of $\Lambda_{\downarrow}(t)$ and $\Lambda_{\uparrow}(t)$, and the consequent opposite sign of the powers $P_{\downarrow}(t)$ and $P_{\uparrow}(t)$. This means that the contributions of different spin to the total power do not separately dissipate heat in the form of a Joule law, as is the case of the unpolarized quantum dot but they can be decomposed as $P_{\sigma}(t)=\xi_{\sigma} P_{\mathrm{ex}}(t)+P_{\mathrm{Joule}, \sigma}(t)$, with $\xi_{\sigma}= \pm$. Here, the component $P_{\mathrm{Joule}, \sigma}(t)$ is associated to the energy dissipated in the form of heat. Instead, $P_{\mathrm{ex}}(t)$ is associated to energy that is transferred in the form of work done by the electrons with the minority spin component on the electrons with the majority spin component or vice versa. The total dissipated power is given by the addition of the Joule components, which is shown in the lower panels of the figure. The mechanism of energy exchange leading to $P_{\mathrm{ex}}(t)$ is a consequence of the combined effect of many-body interactions and the spin polarization due to the magnetic field. In fact, we stress that in the non-interacting case with $U=0, P_{\mathrm{ex}}(t)=0$, as shown in Section III B.

To understand the fundamental difference between the noninteracting and interacting case, let us notice that in the non-

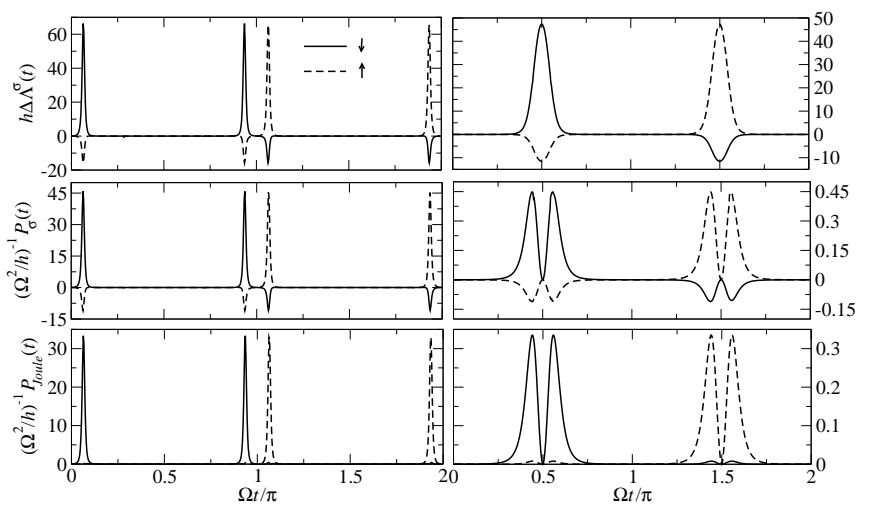

FIG. 9. (Color online) Top panels: Functions $\Lambda^{\sigma}(t)$. Middle panels: Power developed by the forces induced by electrons with spin $\sigma, P_{\sigma}(t)$, Lower panels: Joule power $P_{\text {Joule, } \sigma}(t)$ (see text). Solid and dashed lines correspond to $\sigma=\downarrow$, , respectively. Left (right) panels correspond to $U=0.01 D(U=0.05 D)$, respectively. Other parameters are the same as in Fig. 7

interacting case, electrons with the two spin components behave independently one another. Due to the Zeeman splitting there is one energy level for electrons with $\uparrow$ spin and one for electrons with $\downarrow$ spin, which are rigidly shifted upwards and downwards in energy as the gate voltage changes. Every time that one of these levels gets aligned with the Fermi energy of the reservoir, the occupancy of the quantum dot changes and a current sets between the quantum dot and the reservoir. Such process is accompanied by Joule heating in the form of $P_{\mathrm{Joule}, \sigma}(t)$ with resistance $R_{0}$. Instead, in the interacting regime, the single occupancy is dominated by spins aligned with the magnetic field, while in the configurations with 0 and 2 electrons in the quantum dot, both spin orientations are equally populated. For this reason, when the occupancy changes from singly to double occupancy, there is a flux of spins oriented opposed to the magnetic field following the gate voltage, along with a smaller counter flow of electrons aligned with the magnetic field against the gate voltage. Analogous situations take place when the configuration changes from double to single occupancy and from single occupancy to the empty configuration. The energy to generate the current of the electrons with one of the spin components that opposes to the direction dictated by the external gate voltage is provided by the electrons with the opposite spin component. This is precisely what we have discussed within the RPA approximation in Section IV. C. In a full cycle, this energy exchange averages to zero and only the Joule dissipation remains.

In Fig. 10 we represent the average power over the cycle for a given spin $\bar{P}_{\sigma}$. As a consequence of the symmetry transformation Eq. 27) for the chosen parameters, $\bar{P}_{\downarrow}=\bar{P}_{\uparrow}$. We also represent in the figure the components $\bar{P}_{\uparrow \uparrow}$ and $\bar{P}_{\uparrow \downarrow}$, which correspond to the contributions of the same and opposite spin to the average total power for spin up, according to Eqs. (9), (10) and (11). One can see that the crossed component $\bar{P}_{\uparrow \downarrow}$, which vanishes for $U=0$, decreases rapidly as $U$ is turned on and saturates when $U$ reaches values much larger than both $\Delta$ and the Zeeman splitting $\delta_{Z}$. Instead, for small $U, \bar{P}_{\uparrow \uparrow}$ in- 


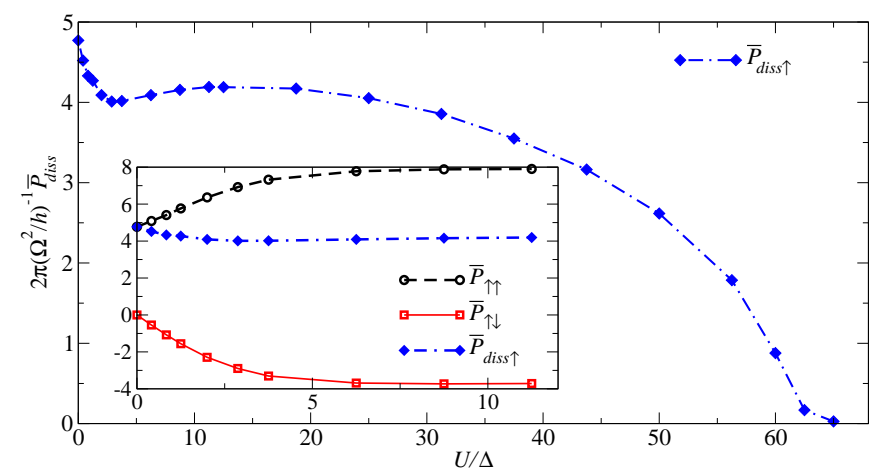

FIG. 10. (Color online) Power developed by the forces induced by electrons with spin $\sigma$ averaged over the cycle as a function of $U$. The inset denotes the different components (see text) for small $U$. Other parameters are the same as in Fig. 7

creases but nos so fast s the decrease in $\bar{P}_{\uparrow \downarrow}$, so that the sum $\bar{P}_{\uparrow}$ decreases for small $U$.

For larger values of $U$ after a modest increase, $\bar{P}_{\sigma}$ decreases because the charge-transfer peaks in the spectral density (separated by $U$ ) cross the Fermi level with a smaller speed, so that the factor $\dot{V}_{g}(t)^{2}$ is smaller [see Eq. [20] ] and when $U$ becomes larger than $2 V_{0}$, the charge-transfer peaks cannot cross the Fermi energy during the cycle and the power drops to zero.

\section{CONCLUSIONS}

We have generalized the theory of the dynamic charge and energy transport of an interacting quantum dot modeled by an Anderson model, coupled to a reservoir and driven by an ac gate voltage in the non-linear adiabatic regime. We considered the cases with and without spin polarization due to a Zeeman splitting. We have shown that the exact adiabatic dynamics is fully characterized by the behavior of the charge susceptibility of the frozen system. We have presented analytical an numerical calculations obtained with NRG and BA techniques.

We have shown that, within and beyond linear response, and zero-temperature the energy is instantaneously dissipated in the form of an instantaneous Joule law with a universal coefficient $R_{0}=e^{2} /(2 h)$ for each spin spin channel. In the case of vanishing magnetic field or in the absence of electron-electron interaction there is a one-to-one correspondence between the dynamics of the driven quantum system and that of a classical circuit sketched in Fig. 22 with $R_{0}$ in each branch. In those cases, electrons with a given spin orientation independently dissipate energy as in the circuit of that figure. However, in the interacting and spin-polarized case, an exchange of energy takes place between electrons with different spin components, which cannot properly accounted by this simple classical circuit. This exchange of power is in some sense akin to the one between driving forces in quantum pumps discussed in Ref. 20. However in that work, that mechanism takes place in non-interacting electrons driven by two time-dependent parameters. Instead, in the present case, it is a consequence of electron-electron interactions in combination with Zeeman splitting in a system driven by a single parameter. The forces involved are non-conservative and proportional to $\dot{V}_{g}(t)$, like the Lorentz forces discussed in Ref. 25. However, in the present case, the energy exchange takes place only at time intervals and do not lead to net work production when averaged over a cycle.

These predictions could be experimentally confirmed in quantum capacitors, where, so far, only the dynamics of the charge transport has been addressed ${ }^{1-3}$. In fact, the Kondo regime has been realized in similar setups without driving 37,38. Hence, the combinations of the experimental arrays of Refs. 1- 3 and Refs. 37 and 38 with suitable fast thermometry as in Refs. 39 and 40 should enable the measurement of the concomitant heat generation.

The charge susceptibility has been measured recently in a quantum dot inside an optical cavity in the Kondo regime. ${ }^{32}$ Our setup provides an alternative way to study this quantity. Furthermore our works predicts non trivial results for the spin resolved components in the presence of strong interactions.

\section{ACKNOWLEDGMENTS}

J.R would like to thank Rok Žitko for his valuable assistance in using the NRG Ljubljana code as well as E. Vernek for discussions. LA thanks M. Moskalets, D. Sanchez and M. Filippone for useful discussions, as well as the hospitality of the Dahlem Center for Complex Quantum Systems, FU-Berlin under the support of the Alexander von Humboldt Foundation and the hospitality of the ICTP-Trieste under the support of a Simons associateship. JR, PR, AAA and LA acknowledge financial support from CONICET, MINCyT and UBACyT from Argentina. AAA sponsored by PIP 112-20110100832 of CONICET and PICT 2013-1045 of the ANPCyT.

\section{Appendix A: Adiabatic Response Formalism}

For completeness we review the treatment presented in Ref. 24. Taking into account that the time-dependent perturbation $\mathcal{V}_{g}(t) n_{d}$ changes slowly in time, expanding $H\left(t^{\prime}\right)$ around $t^{\prime}=$ $t$, the evolution operator is approximated up to linear order in $\dot{\mathcal{V}}_{g}(t)$ as follows

$$
\hat{U}\left(t, t_{0}\right) \approx T \exp \left\{-i H_{t}\left(t-t_{0}\right)-i \int_{t_{0}}^{t} d t^{\prime}\left(t-t^{\prime}\right) F \dot{V}_{g}(t)\right\}
$$

where $F=-\partial H(t) / \partial \mathcal{V}_{g}(t)$ is the "generalized force" induced by the driving and $H_{t}$ is the full Hamiltonian of the system frozen at time $t$.

Hence, to first order in $\dot{V}_{g}(t)$, using $\mathcal{V}_{g}(t)=e V_{g}(t)$, the expectation value of an observable $A$ can be expressed in this "adiabatic approximation" as:

$$
\langle A(t)\rangle \approx\langle A\rangle_{t}-\frac{i e}{\hbar} \int_{t_{0}}^{t}\left(t-t^{\prime}\right)\left\langle\left[A(t), F\left(t^{\prime}\right)\right]\right\rangle_{t} d t^{\prime} \dot{V}_{g}(t)
$$

with $\langle A\rangle_{t}=\operatorname{Tr}\left[\rho_{t} A\right]$, and the operators $F(t), \quad A(t)$ evolving according to the Heisenberg picture with respect to $H_{t}$, 
i.e., $o(t)=e^{\frac{i H_{t} t}{\hbar}} o e^{-\frac{i H_{t} t}{\hbar}}$. From Eq. $\mathrm{A} 2$, and using $F(t)=$ $-n_{d}(t)$, an adiabatic retarded susceptibility corresponding to the frozen Hamiltonian $H_{t}$ can be defined as $\chi_{t}^{A}\left(t-t^{\prime}\right)=$ $-i \theta\left(t-t^{\prime}\right)\left\langle\left[A(t), n_{d}\left(t^{\prime}\right)\right]\right\rangle_{t}$. Hence,

$$
\langle A(t)\rangle \approx\langle A\rangle_{t}+e \Lambda_{t} \dot{V}_{g}(t),
$$

with

$$
\Lambda_{t}=-\frac{1}{\hbar} \int_{t_{0}}^{\infty}\left(t-t^{\prime}\right) \chi_{t}^{A}\left(t-t^{\prime}\right) d t^{\prime}
$$

In Eq. A4 let us make the change of variables $\tau=t-t^{\prime}$. We have, taking $t_{0}=-\infty$,

$$
\Lambda_{t}=-\frac{1}{\hbar} \int_{-\infty}^{\infty} \tau \chi_{t}^{A}(\tau) d \tau
$$

We define the Fourier transform with respect to $\tau=t-t^{\prime}$

$$
\chi_{t}^{A}(\omega)=\int d \tau e^{i \omega \tau} \chi_{t}^{A}(\tau)
$$

We can now use

$$
\frac{\partial \chi_{t}^{A}(\omega)}{\partial \omega}=i \int d \tau e^{i \omega \tau} \tau \chi_{t}^{A}(\tau)
$$

along with the fact that $\Lambda_{t}$ is a real function to write

$$
\Lambda_{t}=-\left.\frac{1}{\hbar} \frac{d \operatorname{Im}\left[\chi_{t}^{A}(\omega)\right]}{d \omega}\right|_{\omega=0}=-\frac{1}{\hbar} \lim _{\omega \rightarrow 0} \frac{\operatorname{Im}\left[\chi_{t}^{A}(\omega)\right]}{\omega}
$$

where in the last step we have assumed that $\operatorname{Im}\left[\chi_{A}(0]=0\right.$. Notice that $\omega$ has units of frequency, thus in order to compare with NRG results one has to transform to energy units by making $\omega=\epsilon / \hbar$.

\section{Appendix B: Equation for the non-linear circuit}

Here we analyze the current through each branch of the classical circuit of Fig. 2] We define the differencial capacitance from

$$
C_{\sigma}\left(V_{C}^{\sigma}\right)=\frac{d q_{\sigma}}{d V_{C}^{\sigma}}
$$

where $q_{\sigma}$ is the charge in the capacitor and $V_{C}^{\sigma}$ the potential drop through it. The total potential drop is $V=V_{C}^{\sigma}+V_{R}^{\sigma}$, where $V_{R}^{\sigma}=R_{\sigma} I_{\sigma}$ is the potential drop through the resistance and $I_{\sigma}$ is the current in the branch with spin $\sigma$. Using $I_{\sigma}=$ $d q_{\sigma} / d t=\dot{q}_{\sigma}$ and Eq. (B1) one has a differential equation for the current,

$$
I_{\sigma}=C_{\sigma}\left(V-R_{\sigma} I_{\sigma}\right)\left[\dot{V}-\frac{d\left(R_{\sigma} I_{\sigma}\right)}{d t}\right] .
$$

The derivatives with respect to the time introduce a factor of the order of the frequency $\Omega$. We now assume small frequencies, specifically $\Omega R_{\sigma} I_{\sigma} \ll 1$. Then as a first approximation, the last term of Eq. (B2) can be neglected. This limit also implies $V \gg R_{\sigma} I_{\sigma}$, an thus to the same order of approximation one can evaluate take $C_{\sigma}(V)$ as the first factor of Eq. (B2) leading to

$$
I_{\sigma}^{1}=C_{\sigma}(V) \dot{V}
$$

to first order in $\dot{V}$. Replacing this result in the second member of Eq. (B2), expanding the first factor and using $d C_{\sigma} / d t=$ $\dot{V} d C_{\sigma} / d V$. we obtain the following expression for the current to second order in $\dot{V}$,

$$
I_{\sigma}=C_{\sigma} \dot{V}-\frac{d\left(R_{\sigma} C_{\sigma}^{2} \dot{V}\right)}{d t}
$$

In order to map the classical circuit to our quantum problem, we identify $V=V_{g}$ as minus the gate voltage of the quantum dot (the energy of electrons with negative charge in the dot changes as the gate voltage increases) and according to the definition Eq. (4) $I_{\sigma}=d q_{\sigma} / d t=-I_{C, \sigma}$. Performing the corresponding replacements one obtains Eq. (12).

\section{Appendix C: Numerics}

In this work we use the known "NRG Ljubljana" free code to calculate the observables of interest (and their correlations) in the case where there is a local Coulomb interaction in the $\operatorname{dot}(U>0)$ and also for $U=0$. There are some subtleties in the NRG method, most of them described in the literature (see for instance Ref. 35 and references therein). However, for the sake of completeness, the main steps of the NRG approach we have employed are described below.

1) We first map the Anderson model onto a Wilson chain, composed of an impurity site with its many body term plus a linear chain of non-interacting sites. Upon this process, the continuous Anderson model has been discretized in the energy space. Here we used the known $z$-trick logarithmic discretization scheme, in which the characteristic energy scale is given by $30,33,34$

$$
\epsilon_{N}=\frac{1-\lambda^{-1}}{\log \lambda} \lambda^{-(N-1) / 2+1-z},
$$

where here we have set $\lambda=2$ and averaged the results for 32 values of $z \in[0,1]$.

2) We then diagonalize iteratively a series of Hamiltonians, starting from an initial $H_{-1}$ describing just the impurity (containing the many-body term) and increasing it with a noninteracting site of the Wilson chain at each iteration. Hence, at a given iteration we need to diagonalize a Hamiltonian $H_{N}$ describing the impurity plus $N+1$ sites of the chain whose dimension is $(N+2) \times(N+2)$. Because of the rapid increase of the dimension of the Hilbert space along the iterating process, we have to take advantage of the symmetries of the Hamiltonians. Here, Since $H_{N}$ commutes with both the total charge 
$\left(\hat{Q}_{N}\right)$ of chain described by $H_{N}$ and with its total spin operator squared $\left(\hat{S}_{N}^{2}\right)$, we exploit $\mathrm{U}(1) \times \mathrm{SU}(2)$ symmetry. In this way, at the $N$-th NRG iteration we diagonalize an enlarged blockdiagonal $(N+2) \times(N+2)$ Hamiltonian matrix whose sectors are labeled by the quantum number $\left(Q_{N}, S_{N}\right)$, where $Q_{N}$ and $S_{N}$ represents the eigenvalues of $\hat{Q}_{N}$ and $\hat{S}_{N}^{2}$, respectively.

Even though taking advantage of the $\mathrm{U}(1) \times \mathrm{SU}(2)$ is a great improvement, it is not enough to allow us to diagonalize large Wilson chains. To overcome this problem it is necessary to truncate the Hilbert space by discarding states. resolution of In the present calculations we retain states with energy up to $E_{\text {keep }}=2 \epsilon_{N}$, a reasonably good choice to converge the manyparticle eigen-energies to the strong coupling fixed point. i.e., it does not change upon reached the fixed point of the At each iteration we not only diagonalize the Hamiltonians but also calculate all the physical quantities we are interested in.

3) With the matrix elements of the relevant quantities at each iteration, we can calculate the thermodynamic and dynamic quantities, such as the static and dynamic susceptibilities. For the dynamic quantities we have employed the full density matrix (FDM) version of the NRG, which is known to provide a better resolution of the spectral quantities. The energy delta peaks appearing in the dynamic susceptibilities are usually broadened by using various smooth distribution functions 35 . In our case we use a modified broadening kernel $K\left(\epsilon, \epsilon_{j}\right)$ de- fined piecewise by 36

$$
K\left(\epsilon, \epsilon_{j}\right)= \begin{cases}L\left(\epsilon, \epsilon_{j}\right) & \text { if }\left|\epsilon_{j}\right| \geq \epsilon_{0}, \\ G\left(\epsilon, \epsilon_{j}\right)[1-h(\epsilon)] & \text { if }\left|\epsilon_{j}\right| \leq \epsilon_{0},\end{cases}
$$

with

$$
\begin{aligned}
G\left(\epsilon, \epsilon_{j}\right) & =\frac{\theta\left(\epsilon \epsilon_{j}\right)}{\sqrt{\pi} \alpha|\epsilon|} \exp \left[-\ln \left(\frac{\left|\epsilon / \epsilon_{j}\right|}{\alpha}-\gamma\right)^{2}\right], \\
L\left(\epsilon, \epsilon_{j}\right) & =\frac{1}{\sqrt{\pi} \epsilon_{0}} \exp \left[-\ln \left(\frac{|\epsilon| \epsilon_{0} \mid}{\alpha}\right)^{2}\right], \\
h(\epsilon) & =\exp \left[-\ln \left(\frac{|\epsilon| \epsilon_{0} \mid}{\alpha}\right)^{2}\right],
\end{aligned}
$$

where $\alpha$ defines the broadening parameter, $\gamma=\alpha / 4$, and $\omega_{0}$ is an energy threshold that changes the broadening distribution function from a Log-Gaussian to a Gaussian at low energies. In practice, smaller $\alpha$ diminishes NRG over broadening, but leads to non-physical oscillations in the dynamic susceptibilities, which can be reduced by averaging over a convenient number of discretization meshes of the conduction band. The broadening parameter is chosen to be $\alpha=0.02$ and $\epsilon_{0}=10^{-99} \mathrm{D}$.
1 J. Gabelli, G. Fève, J.-M. Berroir, B. Placais, A.Cavanna, E.al,Y. Jin, and D. C. Glattli, Science 313, 499 (2006).

2 G. Fève, A. Mahé, J.-M. Berroir, T. Kontos, B. Placais, C. Glattli, A. Cavanna, B. Etienne, and Y. Jin, Science 316, 1169 (2007).

3 J. Gabelli, G. Féve, J.-M. Berroir, and B. Placais, Rep. Prog. Phys. 75, 126504 (2012).

${ }^{4}$ M. Büttiker, A. Prêtre, and H. Thomas, Phys. Rev. Lett. 70, 4114 (1993).

5 A. Prêtre, H. Thomas, and M. Büttiker, Phys. Rev. B 54, 8130 (1996).

${ }^{6}$ M. Büttiker, H. Thomas, and A. Prêtre, Phys. Lett. A 180, 364 (1993).

7 S. E. Nigg, R. López, and M. Büttiker, Phys. Rev. Lett. 97, 206804 (2006).

8 M. Lee, R. López, M.-S. Choi, T. Jonckheere, and T. Martin, Phys. Rev. B 83, 201304 (2011).

9 C. Mora and K. Le Hur, Nat. Phys. 6, 697 (2010).

${ }^{10}$ M. Filippone, K. Le Hur, and C. Mora, Phys. Rev. Lett. 107, 176601 (2011).

11 M. Filippone, C. Mora, Phys. Rev. B 86, 125311 (2012).

12 M. Moskalets, P. Samuelsson, and M. Büttiker Phys. Rev. Lett. 100, 086601 (2008).

13 S. Okhovskaya, J. Splettstoesser, M. Moskalets, and M. Büttiker, Phys. Rev. Lett. 101, 166802 (2008).

14 J. Splettstoesser, M. Moskalets, and M. Büttiker, Phys. Rev. Lett. 103, 076804 (2009).

15 Géraldine Haack, Michael Moskalets, Markus Büttiker Phys. Rev. B 87, 201302(R) (2013).

${ }^{16}$ O. Kashuba, H. Schoeller, J. Splettstoesser, Eur. Phys. Lett. 98, 57003 (2012).
17 M. I. Alomar, J. S. Lim, and David Sánchez, Phys. Rev. B 94, 165425 (2016).

18 D. Litinski, P. W. Boruwer, and M. Filippone, arXiv:1612.04822.

19 M. F. Ludovico, J. S. Lim, M. Moskalets, L. Arrachea and D. Sánchez, Phys. Rev. B 89, 161306(R) (2014).

${ }^{20}$ L. Arrachea, M. Moskalets and L. Martin-Moreno, Phys. Rev. B 75245420 (2007).

${ }^{21}$ M. F. Ludovico, M. Moskalets, D. Sánchez, and L. Arrachea, Phys. Rev. B 94, 035436 (2016).

22 Notice that this definition is consistent with $\dot{U}=\dot{Q}-P$, where $U$ is the internal energy of the electron system (assumed to remain constant for the full system containing the dot and the reservoir).

23 A. C. Hewson, "The Kondo Problem to Heavy Fermions" (Cambridge University Press, Cambridge, 1993).

${ }^{24}$ M.F. Ludovico, F. Battista, F. von Oppen and L. Arrachea, Phys. Rev. B 93, 075136 (2016).

25 J-T. Lü, M. Brandbyge and P. Hedegard, Nano Lett. 101657 (2010).

26 H. Shiba, Prog. of Theor. Phys. 54, 967 (1975).

27 L. Arrachea, Phys. Rev. B 72, 125349 (2005); ibid 75, 035319 (2007).

28 P. B Wiegmann and A. M. Tsvelick, J. Phys. C 16, 2281 (1983).

29 I. J. Hamad, P. Roura-Bas, A. A. Aligia, and E. V. Anda, Physica Status Solidi (b) 253, 478 (2015).

30 R. Žitko, NRG LJUBLJANA, open source numerical renormalization group code, http://nrgljubljana.ijs.si

31 A. A. Aligia, P. Roura-Bas, and S. Florens, Phys. Rev. B 92, 035404 (2015); references therein.

${ }^{32}$ M. M. Desjardins, J. J. Viennot, M. C. Dartiailh, L. E. Bruhat, M. R. Delbecq, M. Lee, M.-S. Choi, A. Cottet, and T. Kontos, Nature 
545, 71 (2017).

33 V.L. Campo and L.N. Oliveira, Phys. Rev. B 72, 104432 (2005).

34 M. Yoshida, M. A. Whitaker, and L. N. Oliveira, Phys. Rev. B 41, 9403 (1990).

35 R. Bulla, T. A. Costi, and T. Pruschke, Rev. Mod. Phys. 80, 395 (2008).

36 Z. Osolin, and R. Žitko, Physical Review B 87, 245135 (2013).

${ }^{37}$ M. Kataoka, C. J. B. Ford, M. Y. Simmons, and D. A. Ritchie
Phys. Rev. Lett. 89, 226803 (2002).

${ }^{38}$ H.-S. Sim, M. Kataoka, C.J.B. Ford, Phys. Rep. 456, 127 (2008).

39 S. Gasparinetti, K. L. Viisanen, O.-P. Saira, T. Faivre, M. Arzeo, M. Meschke, and J. P. Pekola Phys. Rev. Applied 3, 014007 (2015).

${ }^{40}$ M. Zgirski, M. Foltyn, A. Savin, M. Meschke, J. Pekola, arXiv:1704.04762 